\title{
Krankheitsverlauf beim mRCC mit wenig Nebenwirkungen stabil halten
}

— Jeder vierte Patient mit Nierenzellkrebs hat zum Zeitpunkt der Diagnosestellung bereits Metastasen entwickelt. Während die Prognose früher als infaust galt, ermöglichen heute neue zielgerichtete Medikamente, wie beispielsweise der MultikinaseInhibitor Pazopanib, eine deutlich längere Überlebenszeit.

Wie PD Dr. Margitta Retz, München, ausführte, wurde der im Juni 2010 zugelassene Wirkstoff, der einen wichtigen Signalweg für die Gefäßversorgung des Tumors blockiert, für die Erst- und Zweitlinientherapie des metastasierten Nierenzellkarzinoms (mRCC) in die europäischen Leitlinien aufgenommen.
In der zulassungsrelevanten Studie von Sternberg J et al. [J Clin Oncol 2010; 28: 1061-8] hatte sich bei Patienten mit lokal fortgeschrittenem oder metastasiertem Nierenzellkarzinom ( $n=435)$ unter Pazopanib (Votrient ${ }^{\oplus}, 800 \mathrm{mg}$ oral einmal täglich) eine im Placebovergleich signifikante Verlängerung der medianen progressionsfreien Überlebenszeit gezeigt (9,2 vs. 4,2 Monate in der gesamten Teilnehmergruppe, 11,1 vs. 2,8 Monate bei nicht vorbehandelten Patienten). "Ein echter Erfolg", urteilte die Onkologin. Die besonderen Vorteile des Angiogenesehemmers liegen in der besseren Verträglichkeit, betonte Retz. Denn die Kunst des behandelnden Arztes bestehe vor allem darin, den
Krankheitsverlauf mit relativ wenig Nebenwirkungen einigermaßen stabil zu halten. Unerwünschte Begleiterscheinungen - am häufigsten Diarrhö, Erbrechen, Hypertonie, Fatigue und Blutbildveränderungen - seien unter Pazopanib eher milde verlaufen. Nur selten beobachte man schmerzhafte Stomatitiden und toxische Hand-Fuß-Syndrome, wie sie von anderen Target-Therapeutika bekannt sind. Dr. Martina-Jasmin Utzt

Fachpresse-Workshop „Onkologie“, München, 18. Mai 2011

Veranstalter: Amgen, München;

GlaxoSmithKline, München;

Mundipharma, Limburg/Lahn

\section{Prostatakarzinom: Behandlungs- konzept individuell ausrichten}

- Mit einem Anteil von mehr als $25 \%$ ist das Prostatakarzinom (PCa) die häufigste Krebserkrankung des Mannes. Was ist heute bereits therapeutisch machbar? Und welche neuen Optionen sind zu erwarten?

An erster Stelle des lokal begrenzten PCa steht nach wie vor die radikale Prostatektomie, berichtet Prof. Jürgen Gschwend, München. Sie ziele auf eine bestmögliche Tumorkontrolle bei optimaler Erhaltung von Kontinenz und Potenz. Ob man hierbei die offene Technik mit intraoperativem Schnellschnitt-Monitoring oder den minimalinvasiven roboterassistierten Eingriff wählt, hält er für weniger entscheidend als die individuelle Erfahrung und die besonderen "surgical skills" des Operateurs. Als Alternativen zur Operation nannte er die Strahlentherapie und die sogenannte „active surveillance“ mit regelmäßigen Tast-, PSA- und Biopsiekontrollen. „Wichtig ist, das jeweilige Prozedere auf den einzelnen Patienten zuzuschneiden", betonte Gschwend.

Bei ausgedehnteren Tumoren können Operation und adjuvante Strahlentherapie kombiniert werden. Liegen bereits Fernmetastasen (mPC) vor, gilt die antiandrogene Hormontherapie (ggf. intermittierend) als Goldstandard. Eingesetzt werden Agonisten und Antagonisten des luteinisierenden Releasing-Hormons (LHRH) sowie nicht steroidale Antiandrogene wie Bicalutamid.,Entwickelt sich ein kastrationsresistenter Tumor (mCRPC), müssen wir auf Zytostatika wie Docetaxel zurückgreifen", so Gschwend.

Dr. Martina-Jasmin Utzt

Journalistenworkshop „In der Diskussion: Die Therapie des Prostatakarzinoms: Was ist heute möglich - was können wir künftig erwarten?", München, 18. Mai 2011

Veranstalter: Janssen-Cilag, Neuss

\section{Gesucht: aktive Männergesundheitspraxis}

- Die Deutsche Gesellschaft für Mann und Gesundheit e.V. (DGMG) zeichnet dieses Jahr zum zweiten Mal Arztpraxen aus, die sich besonders um die Gesundheit der Männer kümmern. Im vergangenen Jahr wurden erstmals drei Praxen prämiert, die sich durch Aktivitäten für die Männergesundheit auszeichneten. „Das war die wirksamste und beste Marketing-Aktion“, meint Vorjahresgewinner Dr. Michael Blessing, Urologe aus Bad Wörishofen. Bewertet werden zum Beispiel Angebote wie besondere (Abend-) Sprechstunden für Männer, spezielle Vorsorgeuntersuchungen oder auch das Auslegen von "Männer-Zeitschriften“ im Wartezimmer. Auch Flyer oder Plakate, die beispielsweise auf Vorsorgeuntersuchungen für Männer hinweisen, fließen in die Bewertung mit ein. Patienten-Veranstaltungen zum Thema Männergesundheit sind ebenfalls ein sinnvolles Instrument, um das Gesundheitsbewusstsein der Männer zu schärfen. Interessierte Praxen können sich online bewerben unter www.mann-und-gesundheit.com. Unterstützt wird die Aktion von der Apogepha Arzneimittel GmbH. Die drei besten Praxen können in diesem Jahr ein Praxis-PR-Paket mit Flyern und Postern für das Wartezimmer, eine Broschüre über Praxis-Marketing und PR sowie einen Pressetext für die regionale Presse über die Auszeichnung gewinnen.

Die Sieger werdem am 2. November 2011 in Bad Homburg feierlich ausgezeichnet. An diesem Tag wird auch der Gesundheitsmann 2011 in Bad Homburg gekürt. Nominiert sind Markus Lanz, Frank Schätzing, Peyman Amin, Heiner Brand, Sven Hannawald, Marco Huck, Joey Kelly und Heiner Lauterbach. Gewählt wird im September. Näheres wird zeitnah auf die Homepage der DGMG gestellt.

Nach Informationen der DGMG, Bad Homburg 